\title{
Mixed reality models based on low-dose computed tomography technology in nephron-sparing surgery are better than models based on normal-dose computed tomography
}

\author{
Guan $\mathrm{Li}^{1}$, Zhiqiang $\mathrm{Cao}^{2}$, Jinbao Wang ${ }^{3}$, Xin Zhang ${ }^{4}$, Longjiang Zhang ${ }^{1}$, Jie Dong ${ }^{5}$, Guangming Lu ${ }^{1}$ \\ ${ }^{1}$ Department of Medical Imaging, Jinling Hospital, Medical School of Nanjing University, Nanjing, China; ${ }^{2}$ Department of Urology, General \\ Hospital of Northern Theater Command, Shenyang, China; ${ }^{3}$ Department of Radiology, General Hospital of Northern Theater Command, \\ Shenyang, China; ${ }^{4}$ Department of Radiology, The First Affiliated Hospital of China Medical University, Shenyang, China; ${ }^{5}$ Department of Urology, \\ Jinling Hospital, Medical School of Nanjing University, Nanjing, China
}

Correspondence to: Jie Dong. Department of Urology, Jinling Hospital, Medical School of Nanjing University, 305 Eastern Zhongshan Road, Nanjing 210002, China. Email: smiledongjie@163.com; Guangming Lu. Department of Medical Imaging, Jinling Hospital, Medical School of Nanjing University, 305 Eastern Zhongshan Road, Nanjing 210002, China. Email: cjr.luguangming@vip.163.com.

Background: Nephron-sparing surgery has been widely applied in the treatment of renal tumors. Previous studies have confirmed the advantages of mixed reality technology in surgery. The study aimed to explore the optimization of mixed reality technology and its application value in nephron-sparing surgery.

Methods: In this prospective study of 150 patients with complex renal tumors (RENAL nephrometry score 27 ) who underwent nephron-sparing surgery, patients were randomly divided into Group A (the normal-dose mixed reality group, n=50), Group B (the low-dose mixed reality group, n=50), and Group C (the traditional computed tomography image group, $\mathrm{n}=50$ ). Group A and Group $\mathrm{C}$ received the normaldose computed tomography scan protocol: $120 \mathrm{kVp}, 400 \mathrm{~mA}$, and $350 \mathrm{mgI} / \mathrm{mL}$, while Group B received the low-dose computed tomography scan protocol: $80 \mathrm{kVp}$, automatic tube current modulation, and $320 \mathrm{mgI} / \mathrm{mL}$. All computed tomography data were transmitted to a three-dimensional visualization workstation and underwent modeling and mixed reality imaging. Two senior surgeons evaluated mixed reality quality. Objective indexes and perioperative indexes were calculated and compared.

Results: Compared with Group A, the radiation effective dose in Group B was decreased by 39.6\%. The subjective scores of mixed reality quality in Group B were significantly higher than those of Group A $(\mathrm{Z}=-4.186, \mathrm{P}<0.001)$. The inter-observer agreement between the two senior surgeons in mixed reality quality was excellent $(\mathrm{K}=0.840, \mathrm{P}<0.001)$. The perioperative indexes showed that the mixed reality groups were significantly different from the computed tomography image group (all $\mathrm{P}<0.017$ ). More cases underwent nephron-sparing surgery in the mixed reality groups than in the computed tomography image group $(\mathrm{P}<0.0017)$.

Conclusions: Low-dose computed tomography technology can be effectively applied to mixed reality optimization, reducing the effective dose and improving mixed reality quality. Optimized mixed reality can significantly increase the cases of successful nephron-sparing surgery and improve perioperative indexes.

Keywords: Mixed reality; nephron-sparing surgery (NSS); low-dose; computer tomography; adherent perinephric fat (APF)

Submitted Aug 08, 2020. Accepted for publication Jan 29, 2021.

doi: 10.21037/qims-20-956

View this article at: http://dx.doi.org/10.21037/qims-20-956 


\section{Introduction}

Currently, nephron-sparing surgery (NSS) is widely applied in treating renal tumors (1). For $\geq$ stage T1b or complex renal tumors (RENAL nephrometry score $\geq 7$ ), NSS should be selected according to the location, shape, and relationship with adjacent regions (2). Mixed reality (MR) is a new generation of visualization technology, which facilitates an immersive interaction between the user, the real scene, and the virtual scene (3). Preoperatively, MR can help formulate accurate operation plans and reduce the difficulty in identifying complex anatomical structures of tumors. During surgery, MR can display the renal tumor in real-time, which helps locate the renal tumor quickly and accurately (4).

Adequate display of the renal tumor characteristics and the peritumoral situation is key to successful NSS (5). When adherent perinephric fat (APF) occurs around the renal tumor, it can directly affect the success rate of NSS. APF is a chronic inflammatory response induced by perinephric fat, leading to fibrous tissue proliferation, adhesion, and fat properties changes. Related studies have reported that the presence of APF can increase surgical complexity and extend operative times, and can even lead to forced radical nephrectomy (RN) (6). With the development of individualized medicine, it is a challenge to display APF under MR. In the present study, we first tried to perform an MR display of the APF region.

MR imaging is based on computed tomography (CT) DICOM data (7). The quality of CT data directly affects the quality of MR. To obtain high-quality MR, higher tube voltage, tube current, or contrast medium doses were adopted for CT scanning. However, higher CT radiation doses can damage normal tissues and organs, such as gonads, thyroid, and breast (8), while higher contrast medium doses can lead to contrast-induced nephropathy and hypersensitivity (9). Therefore, this study aimed to investigate the optimization of MR and its application value.

\section{Methods}

\section{Study design and patient selection}

Patients ( $\mathrm{n}=150 ; 83$ males and 67 females, with an age range of 33-84 years and a median age of $58.5 \pm 12.6$ years) with complex renal tumors (RENAL nephrometry score 27 ) (10) who underwent NSS were reviewed prospectively between February 2019 and May 2020. A total of 150 patients were randomly divided into three groups, namely Group
A (the normal-dose MR group, n=50), Group B (the lowdose MR group, $n=50$ ), and Group C (the traditional CT image group, $n=50$ ). Two senior surgeons (completed NSS $\geq 600$ cases) performed all the operations together. For those who could not successfully undergo NSS, RN was selected. According to the operation records and postoperative follow-up, the perioperative indicators were obtained. This study was performed following the Declaration of Helsinki and was approved by the Jinling Hospital's Ethics Committee, Medical School of Nanjing University. All patients gave informed consent to participate in the study before the examination.

The inclusion criteria were as follows: (I) renal artery (RA) computed tomography angiography (CTA) examination was performed before surgery; (II) RENAL nephrometry score $\geq 7$; (III) Mayo adhesive probability (MAP) score >2 (6); (IV) all patients underwent NSS or RN. The exclusion criteria were as follows: (i) contrast medium allergy; (ii) severe cardiovascular disease, cerebrovascular disease, and impaired renal function; (iii) second operation for recurrent renal cell carcinoma (RCC).

\section{CT scanning protocol}

All patients underwent RA CTA examination on a multidetector CT machine (Discovery CT750 HD; GE Healthcare, USA). The CT scanning range was from the bilateral adrenal level to the bilateral lower pole. For Group A and Group C, the CT scan parameters were as follows: tube voltage $120 \mathrm{kVp}$, tube current $400 \mathrm{~mA}$, and the filtered back-projection (FBP) reconstruction algorithm. For Group B, the CT scan parameters were tube voltage $80 \mathrm{kVp}$, automatic tube current modulation (ATCM, 100-400 mA) technology, and the adaptive statistical iterative reconstruction (ASiR) algorithm. Other scanning parameters were applied as follows: 64 detectors with 0.625 mm section thickness; collimation $40 \mathrm{~mm}$; rotation time was $0.5 \mathrm{~s}$; the pitch was $0.984: 1$; image matrix was $512 \times 512$; the field of view was $250 \mathrm{~mm}$; noise index was 11.0; slice thickness was $5 \mathrm{~mm}$, and reconstruction slice thickness was $0.625 \mathrm{~mm}$.

\section{Contrast medium injection scheme}

Group A and Group C were injected with $350 \mathrm{mgI} / \mathrm{mL}$ contrast medium (Omnipaque 350, GE Healthcare, USA), and Group B was injected with $320 \mathrm{mgI} / \mathrm{mL}$ contrast medium (Iodixanol 320, GE Healthcare, USA). 


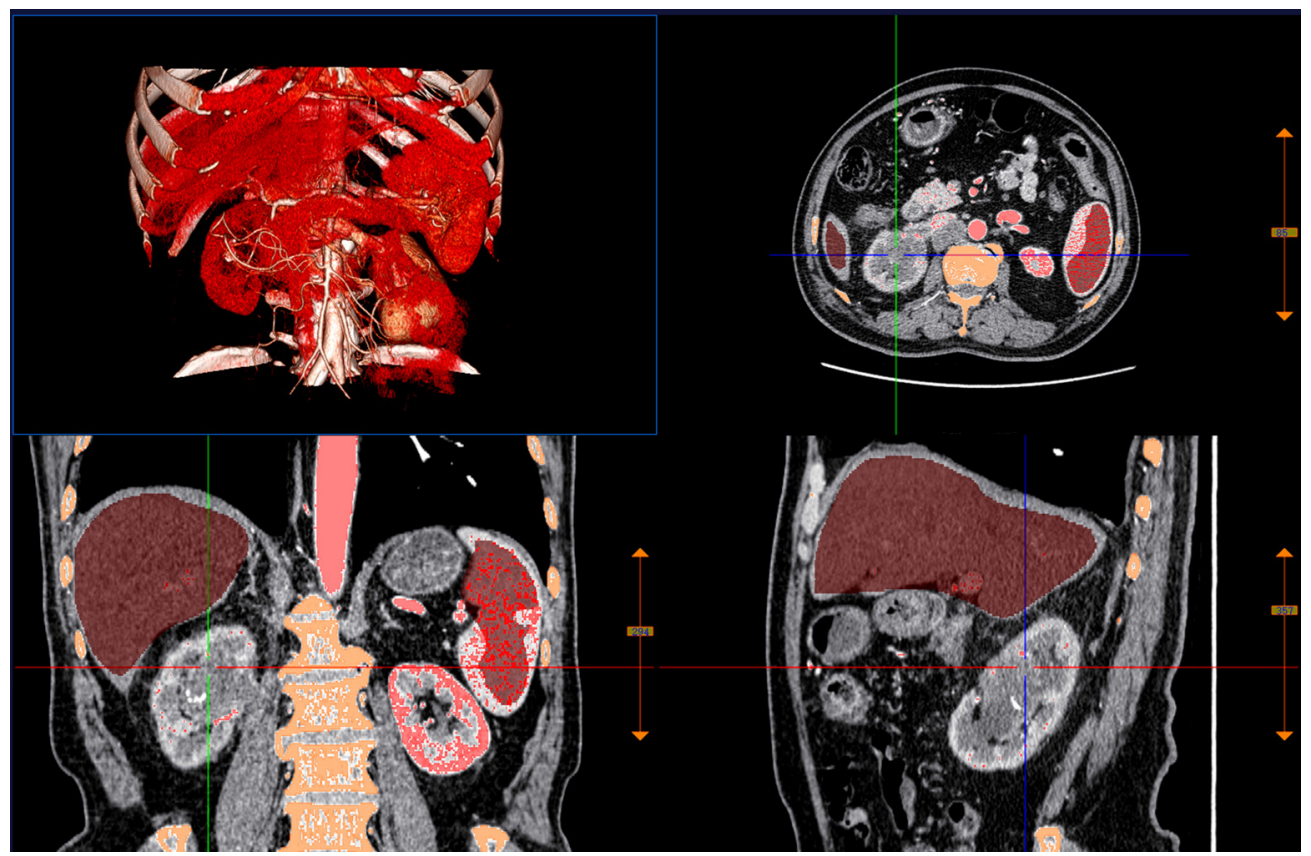

Figure 1 Holographic V3D modeling interface.

The contrast medium dose was approximately $45-75 \mathrm{~mL}$ $(0.9-1.0 \mathrm{~mL} / \mathrm{kg})$, the flow rate was $5 \mathrm{~mL} / \mathrm{s}$, and the injected saline was $30 \mathrm{~mL}$. The contrast medium was injected using a high-pressure syringe (Urich, Medical, USA) into the median cubital vein. Automatic bolus tracking was used, and the abdominal aorta (AA)'s trigger threshold was 150 Hounsfield units (HU).

\section{Radiation dose evaluation}

After CT scanning, the values of the CT dose index volume $\left(\mathrm{CTDI}_{\mathrm{vol}}\right)$ and dose-length product (DLP) were recorded. According to the effective dose (ED) formula, $\mathrm{ED}=\mathrm{DLP} \times \mathrm{K}$, where the conversion factor $\mathrm{K}$ was $0.015 \mathrm{mSv} /(\mathrm{mGy} \cdot \mathrm{cm})$ (11). Due to CT scanning conditions and the fact that contrast medium doses were consistent between Group A and Group C, we selected Group A as the representative group for evaluation.

\section{Holographic MR display}

All DICOM files of Group A and Group B were input into holographic MR software (VISUAL Co., Ltd., Beijing, China). We sequentially used visual edge detection, automatic segmentation extraction, and registration steps to operate on the target. For those that could not be automatically identified, we chose to draw regions of interest (ROIs) manually. Irrelevant or isolated parts were removed at the same time. The kidney, renal vessels, renal collection system, tumor, renal capsule, and APF area were retained. After expansion, corrosion, and smoothing steps, the above target tissues were saved as STL files and then imported into scene editing mode (Figure 1). In this scene mode, we could adjust the color and transparency of target tissues. MR results were imported into MR glasses or directly imported into the laparoscopic video system (Figure 2).

\section{Quantitative evaluation}

CT DICOM data objective quality measurements were performed by one radiologist (J Wang) with 15 years of experience in image post-processing. On the GE AW4.6 workstation, ROIs were selected to ensure the same target tissue. The ROI size of the ROI was defined as $1 / 2$ size larger than the vascular lumen area or $100 \mathrm{~mm}^{2}$ (other parts except for blood vessels), avoiding vascular walls and vascular calcification. The location of the ROI was selected to measure the CT value at the same level as the RA (approximately $1.0 \mathrm{~cm}$ from the beginning of the RA), AA, erector spinae (ES), and air in front of the anterior abdominal wall (Figure 3). The standard deviation 

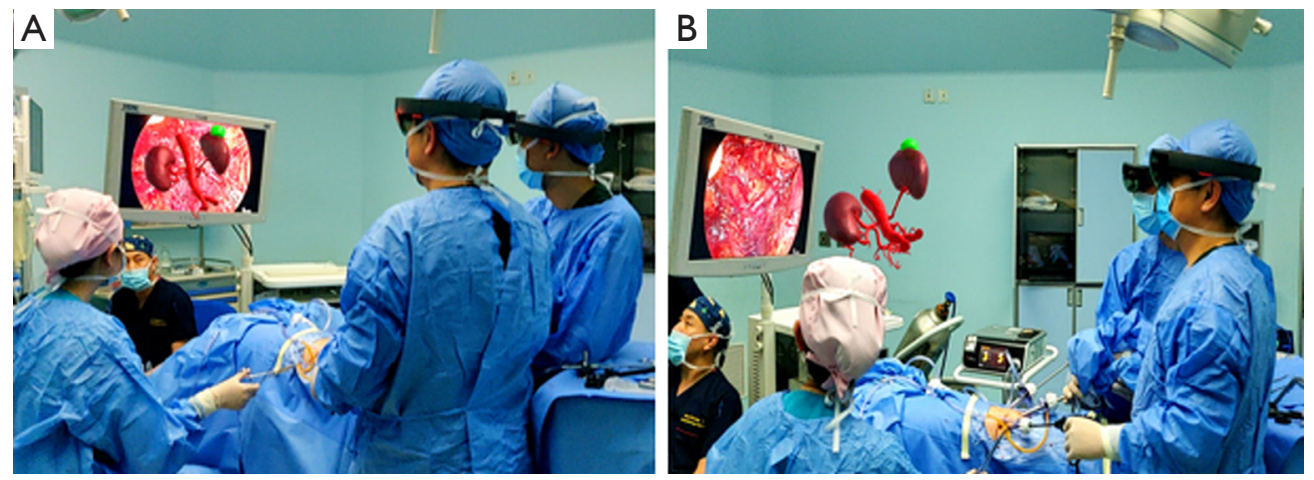

Figure 2 MR display modes. (A) MR display adopted in the laparoscopic video system; (B) MR display adopted in MR glasses. MR, mixed reality.
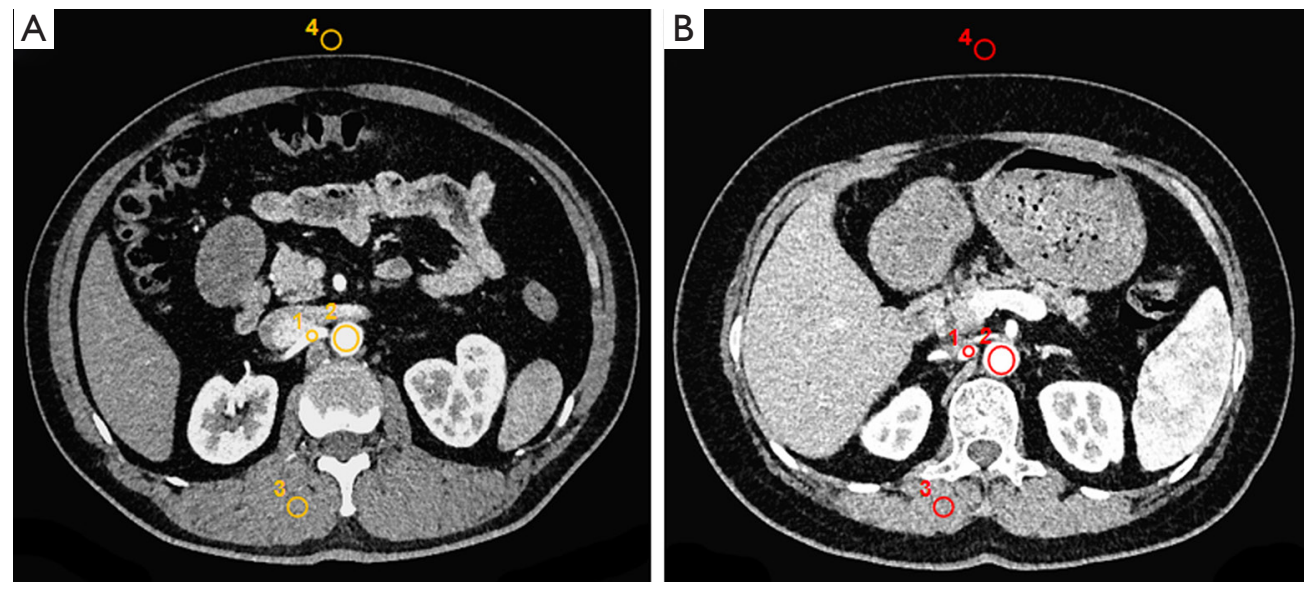

Figure 3 ROI selection of CT DICOM data in Group A and Group B. (A) Male, 46 years, received the normal-dose CT scanning protocol

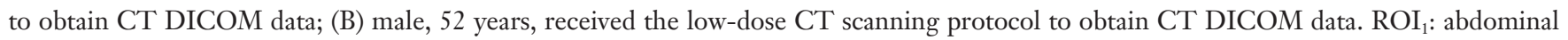
aorta; $\mathrm{ROI}_{2}$ : renal artery; $\mathrm{ROI}_{3}$ : erector spinae; $\mathrm{ROI}_{4}$, air. ROI, region of interest; CT, computed tomography.

(SD) of air in front of the anterior abdominal wall CT values was defined as image noise (IN). Based on the above measurements, the signal-to-noise ratio (SNR) was calculated using the formula: $\mathrm{SNR}=\mathrm{CT}_{\mathrm{RA}} / \mathrm{SD}_{\text {air }}$ and the contrast-noise ratio (CNR) was obtained using the formula: $\mathrm{CNR}=\left(\mathrm{CT}_{\mathrm{RA}}-\mathrm{CT}_{\mathrm{ES}}\right) / \mathrm{SD}_{\text {air }}(12)$.

\section{Qualitative evaluation}

A total of 100 cases (Groups A and B) were separately displayed and independently scored by two senior surgeons (J Dong and Z Cao) who were blinded to the study protocols. Since MR is an emerging technology, there is no authoritative organization to formulate evaluation standards for MR quality. Herein, we referred to the CT DICOM data quality score, the related literature on MR imaging, and surgeons' opinions to formulate the MR quality score $(13,14)$.

MR quality was evaluated on a 4-point scale as follows:

- 4 (excellent), the MR displayed kidney, tumor, renal vessels, renal collection system, and renal capsule as intact and vivid; the size, shape, and location of the tumor and APF region were precise;

* 3 (good), the MR displayed kidney, tumor, renal vessels, renal collection system, and renal capsule as complete and clear; the size, shape, and location of the tumor and APF region were accurate;

* 2 (poor), the MR displayed kidney, tumor, renal 
Table 1 Patient demographics among Groups A, B, and C

\begin{tabular}{lccc}
\hline Variable & Group A $(\mathrm{n}=50)$ & Group B $(\mathrm{n}=50)$ & Group C $(\mathrm{n}=50)$ \\
\hline Mean age (years) & $55.7 \pm 12.7$ & $57.0 \pm 14.3$ & $55.6 \pm 13.4$ \\
Height $(\mathrm{cm})$ & $167.3 \pm 7.7$ & $169.9 \pm 8.0$ & $171.5 \pm 7.2$ \\
Weight $(\mathrm{kg})$ & $75.2 \pm 12.6$ & $73.0 \pm 11.1$ & $79.9 \pm 11.5$ \\
Sex, $\mathrm{n}[\%]$ & & & $37[74]$ \\
Male & $32[64]$ & $35[70]$ & $13[26]$ \\
Female & $18[36]$ & $15[30]$ & $27.2 \pm 4.5$ \\
BMI (kg/m $\left.{ }^{2}\right)$ & $26.8 \pm 4.2$ & $25.9 \pm 4.1$ & $3.0 \pm 1.2$ \\
MAP score & $2.9 \pm 1.0$ & $2.6 \pm 0.9$ & $8.6 \pm 1.6$ \\
RENAL score & $8.2 \pm 1.2$ & $8.4 \pm 1.3$ & 8.6 \\
\hline
\end{tabular}

BMI, body mass index; MAP, Mayo adhesive probability.

vessels, renal collection system, and renal capsule as incomplete and coarse; the size, shape, and location of the tumor and APF region were inaccurate;

* 1 (non-diagnostic), the MR displayed kidney, tumor, renal vessels, renal collection system, and renal capsule as absent and unclear; the size, shape, and location of the tumor and APF region were erroneous.

MR quality of 3 points or more was considered to satisfy the needs of surgeons. In case of inter-observer disagreement, the final decisions were reached by consensus.

\section{Statistical analysis}

SPSS version 17.0 (SPSS Inc. Chicago, IL, USA) was used for statistical analysis. Quantitative data were shown as mean \pm SD. Categorical variables were described as frequencies or percentages. Student's $t$-test was used to compare the means of two independent samples. Bonferroni's adjustment was used to correct for multiple testing. Wilcoxon test was used for the analysis of hierarchical data. Mann-Whitney $U$ tests were used to compare the MR quality scores between the two groups. The $\chi^{2}$ test was used to examine the count data. Correlations were analyzed using the Pearson chi-squared test. The consistency of the evaluation of MR quality by the two senior surgeons was estimated by the Kappa test, which was interpreted as follows: excellent, $\mathrm{K}$ value range was $[0.81,1.00)$; good, $\mathrm{K}$ value range was $[0.61,0.80)$; poor, $\mathrm{K}$ value range was $[0.41,0.60)$; very poor, $\mathrm{K}$ value range was $[0$, $0.40)$. A $P$ value $<0.05$ was statistically significant.

\section{Results}

\section{Study population and demographic characteristics}

Table 1 reveals the demographic characteristics among Groups A, B, and C. No significant differences were found in mean age, weight, height, sex, body mass index (BMI), MAP score, and RENAL score among Groups A, B, and C (all $\mathrm{P}>0.05$ ).

\section{Quantitative analysis}

Table 2 shows the comparative results of relevant CT values, IN, SNR, and CNR between Group A and Group B. The CT values of RA, AA, and ES in Group A (335.4 $\pm 52.7,340.6 \pm 42.4$, and $62.1 \pm 5.3 \mathrm{HU}$, respectively) were significantly lower than those in Group B (553.6 \pm 56.2 , $623.7 \pm 45.1$, and $72.4 \pm 4.8 \mathrm{HU}$, respectively; all $\mathrm{P}<0.001$ ). The CT values of air between Group A and Group B $(-992.8 \pm 3.2 v s .-993.1 \pm 3.8 \mathrm{HU}, \mathrm{P}>0.05)$ were not significantly different. The CNR and SNR in Group B $(43.8 \pm 5.1$ and $49.1 \pm 6.8$, respectively) were significantly higher than those in Group A $(17.5 \pm 5.7$ and 21.6 \pm 6.7 , respectively).

\section{Radiation dose measurement results}

The CTDI ${ }_{\text {vol }}$, DLP, and ED values for Group A were significantly higher than those for Group B $\left(14.3 \pm 1.8\right.$ vs. $9.4 \pm 1.2 \mathrm{mGy}$ for $\mathrm{CTDI}_{\mathrm{vol}} ; 343.2 \pm 35.7$ vs. $225.9 \pm 31.6 \mathrm{mGy}-\mathrm{cm}$ for DLP; $5.3 \pm 1.3$ vs. $3.2 \pm 1.1 \mathrm{mSv}$ 
Table 2 Quantitative analysis between Groups A and B

\begin{tabular}{lccc}
\hline Variable & Group A $(n=50)$ & Group B $(n=50)$ & $P$ \\
\hline RA $(H U)$ & $335.4 \pm 52.7$ & $553.6 \pm 56.2$ & $<0.001$ \\
AA $(\mathrm{HU})$ & $340.6 \pm 42.4$ & $623.7 \pm 45.1$ & $<0.001$ \\
ES $(\mathrm{HU})$ & $62.1 \pm 5.3$ & $72.4 \pm 4.8$ & $<0.001$ \\
Air $(\mathrm{HU})$ & $-992.8 \pm 3.2$ & $-993.1 \pm 3.8$ & 0.670 \\
IN & $16.1 \pm 3.2$ & $11.4 \pm 2.8$ & $<0.001$ \\
CNR & $17.5 \pm 5.7$ & $43.8 \pm 5.1$ & $<0.001$ \\
SNR & $21.6 \pm 6.7$ & $49.1 \pm 6.8$ & $<0.001$ \\
\hline
\end{tabular}

$\mathrm{RA}$, renal artery; AA, abdominal aorta; ES, erector spinae; IN, image noise; CNR, contrast-noise ratio; SNR, signal-to-noise ratio.

Table 3 Comparison of CT radiation dose between Group A and Group B

\begin{tabular}{lccc}
\hline Variable & Group A $(\mathrm{n}=50)$ & Group B $(\mathrm{n}=50)$ & $\mathrm{P}$ \\
\hline CTDI $_{\text {vol }}(\mathrm{mGy})$ & $14.3 \pm 1.8$ & $9.4 \pm 1.2$ & $<0.001$ \\
DLP $(\mathrm{mGy}-\mathrm{cm})$ & $343.2 \pm 35.7$ & $223.2 \pm 31.6$ & $<0.001$ \\
ED $(\mathrm{mSv})$ & $5.3 \pm 1.3$ & $3.2 \pm 1.1$ & $<0.001$ \\
\hline
\end{tabular}

$\mathrm{CTDI}_{\mathrm{vol}}$, CT dose index volume; DLP, dose-length product; ED, effective dose.

Table 4 Subjective scores of MR quality between Group A and Group B

\begin{tabular}{lccccccc}
\hline Groups & 1 score & 2 score & 3 score & 4 score & Total (case) & Z & P \\
\hline Group A & 0 & 0 & 34 & 16 & 50 & -4.186 & $<0.001$ \\
Group B & 0 & 0 & 13 & 37 & 50 & \\
\hline
\end{tabular}

for $\mathrm{ED}$; all $\mathrm{P}<0.001$; Table 3). Compared with Group A, $\mathrm{CTDI}_{\mathrm{vol}}$, DLP, and ED in Group B decreased by $34.3 \%$, $34.2 \%$, and $39.6 \%$, respectively.

\section{Subjective scoring results of mr quality}

The subjective scores of MR quality in Group A and Group $B$ were above 3 points, indicating that they could better meet surgeons' needs. Moreover, the subjective scores of MR quality in Group B were significantly higher than those of Group A. After the Mann-Whitney U test analysis, the subjective scores of MR quality showed a significant difference between Group A and Group B $(Z=-4.186$, $\mathrm{P}<0.001 ;$ Table 4, Figures 4,5). In the 100 cases of MR results, eight cases were inconsistent with the scores of the two senior surgeons. After discussion, three cases finally obtained a score of 3 , and five cases finally obtained a score of 4. The inter-observer agreement between the two senior surgeons in MR quality was very good $(\mathrm{K}=0.840, \mathrm{P}<0.001$; Figure 6).

\section{Perioperative results}

The perioperative indexes of Groups A, B, and C were compared. The results showed that the operation time (OT), warm ischemia time (WIT), and estimated blood loss (EBL) in Groups A and B were significantly less than those in Group C. The number of cases that underwent NSS was greater in Groups A and B than in Group C (54\% vs. 28\%, $58 \%$ vs. $28 \%$, all $\mathrm{P}<0.017)$. OT, WIT, EBL, and the number of cases that underwent NSS between Group A and Group $\mathrm{B}$ were not significantly different (all $\mathrm{P}>0.05$ ). There were no significant differences in other indexes (such as hospital stay, preoperative or postoperative serum creatinine (Scr), and postoperative complications) among the three groups (all P>0.05; Table 5). 

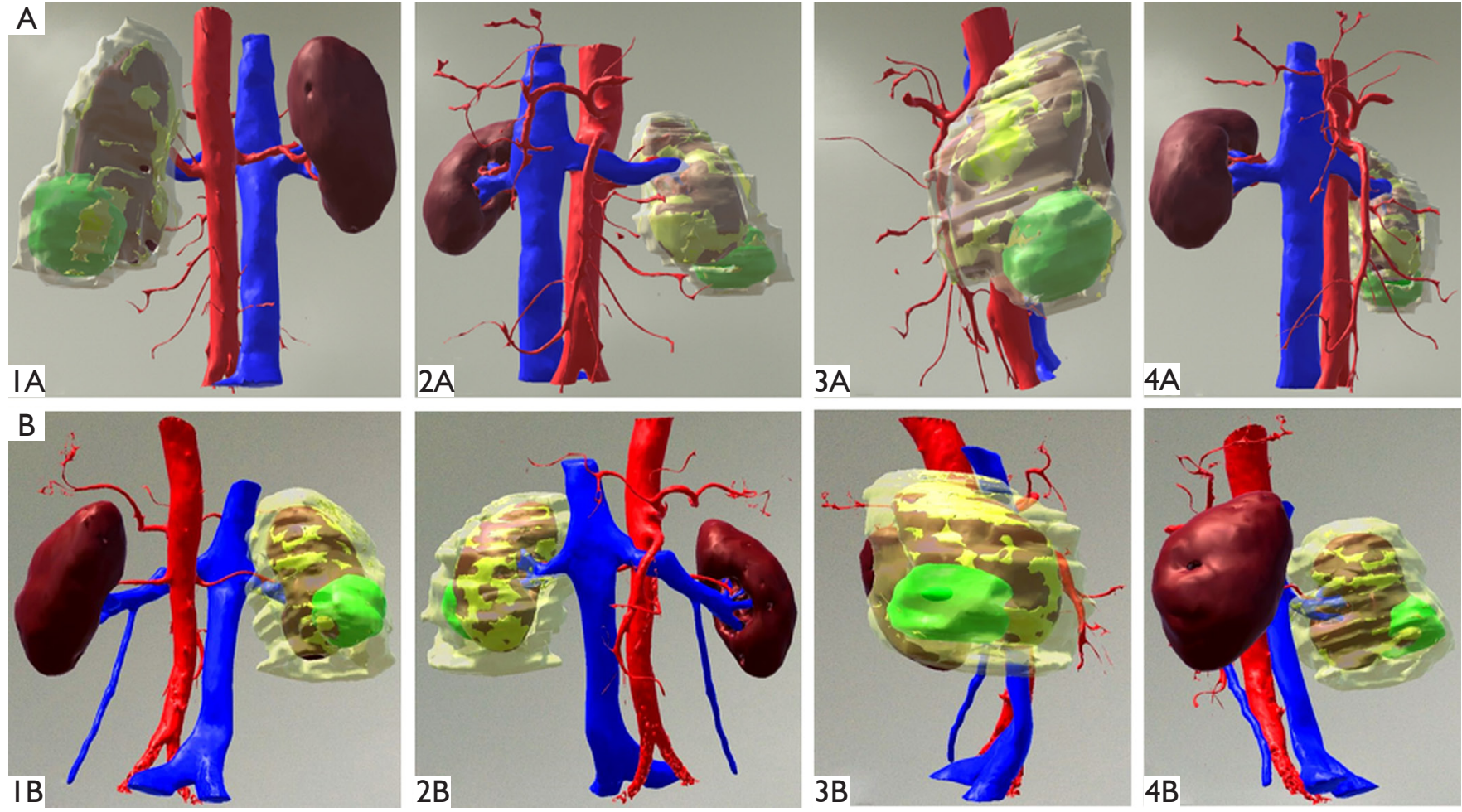

Figure 4 Group A and Group B V3D modeling displays. (A) Male, 46 years, left renal tumor; received the normal-dose protocol and underwent V3D modeling; (B) male, 52 years, right renal tumor, received the optimized protocol and underwent V3D modeling. 1A, 1B: front view; 2A, 2B: posterior view; 3A, 3B: lateral view; 4A, 4B: medial view. Yellow: APF region; green: renal tumor; vermilion: kidney; red: artery; blue: vein.
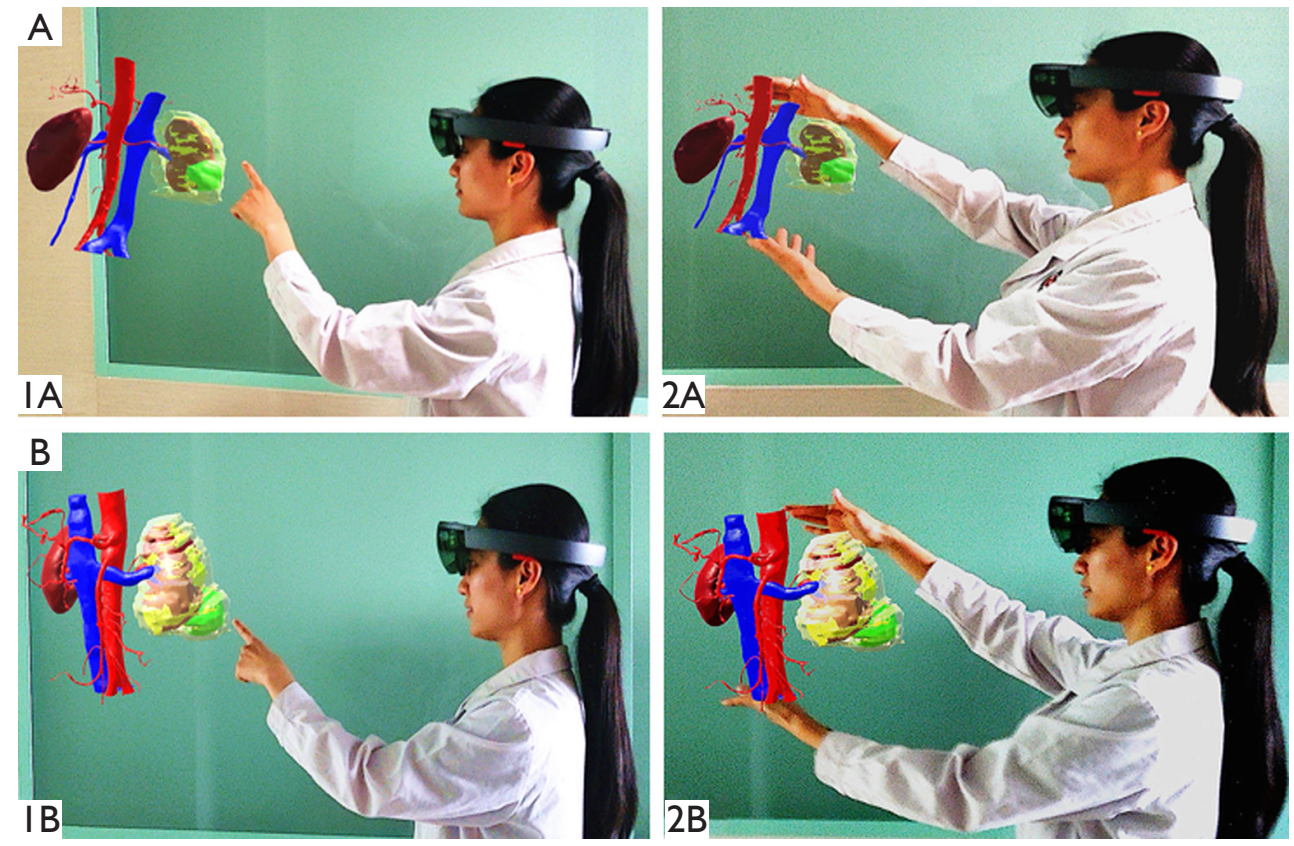

Figure $5 \mathrm{MR}$ displays performed for Group A and Group B. (A) MR display of a male, 46 years, left renal tumor, who received the normaldose protocol; (B) MR display of a male, 52 years, right renal tumor, who received the optimized protocol. 1A, 1B: one hand operation; 2A, 2B: both hands operation. Yellow: APF region; green: renal tumor. 


\begin{tabular}{|l|c|c|c|c|c|c|}
\hline & & \multicolumn{3}{|c|}{ Surgeon B } & \multirow{2}{*}{ K } \\
\hline \multirow{3}{*}{ Surgeon A } & & 3 score & 4 score & Total (case) & \multirow{2}{*}{0.840} \\
\cline { 2 - 7 } & 3 score & 44 & 3 & 47 & $<0.001$ \\
\cline { 2 - 7 } & 4 score & 5 & 48 & 53 & 100 & \\
\hline
\end{tabular}

Figure 6 Consistency of MR quality evaluation by the two senior surgeons. MR, mixed reality.

Table 5 Comparison of perioperative indexes between Groups A, B, and C

\begin{tabular}{|c|c|c|c|}
\hline Variable & Group A $(n=50)$ & Group B $(n=50)$ & Group C $(n=50)$ \\
\hline WIT (min) & $17.5 \pm 3.7$ & $15.7 \pm 2.4$ & $27.4 \pm 4.3^{\star \bullet}$ \\
\hline $\mathrm{EBL}(\mathrm{mL})$ & $23.4 \pm 7.9$ & $20.9 \pm 5.1$ & $43.7 \pm 8.7^{\star \bullet}$ \\
\hline Hospital stay (days) & $6.5 \pm 1.6$ & $6.3 \pm 1.2$ & $6.8 \pm 1.4$ \\
\hline Postoperative Scr ( $\mu \mathrm{mol} / \mathrm{L})$ & $118.3 \pm 17.2$ & $121.8 \pm 16.4$ & $127.3 \pm 14.7$ \\
\hline LPN conversion to LRN, n (\%) & $15[30]$ & $13[26]$ & $21[42]^{\star \bullet}$ \\
\hline Postoperative complications, $\mathrm{n}(\%)$ & $3[6]$ & $5[10]$ & $3[6]$ \\
\hline Underwent NSS, n (\%) & $27[54]$ & 29 [58] & $14[28]^{\star \bullet}$ \\
\hline
\end{tabular}

$P$ value after Bonferroni correction for multiple comparisons $(P=0.05 / 3 \approx 0.017)$. ${ }^{*}$, Group $C$ vs. Group $A, P<0.017 ;{ }^{\bullet}$, Group $C$ vs. Group $\mathrm{B}, \mathrm{P}<0.017$. OT, operation time; WIT, warm ischemia time; EBL, estimated blood loss; Scr, serum creatinine; LPN, laparoscopic partial nephrectomy; LRN, laparoscopic radical nephrectomy; NSS, nephron-sparing surgery.

\section{Discussion}

As a new generation of holographic visualization technology, MR can read image information intuitively, stereoscopically, and comprehensively in the most natural way, which helps surgeons shorten the learning cycle before surgery and quickly locate renal tumors during surgery (3). This study shows that MR can effectively increase successful NSS cases, shorten OT, and decrease WIT and EBL compared with traditional CT imaging. This result is consistent with that of Shirk et al. (15). Shirk et al. proved that $3 \mathrm{D}$ virtual reality (VR) models could shorten the OT, and as the nephrometry scores increased, 3D VR models showed a significant difference from non3D VR models in terms of OT. Hence, our study enrolled patients with nephrometry scores $\geq 7$, omitting patients with low nephrometry scores. We directly concluded a significant difference in OT between the normal-dose/low-dose MR groups and the non-MR group $(\mathrm{P}<0.001)$. Furthermore, we demonstrated that low-dose CT technology could be effectively applied to MR, reducing ED (by approximately 42.3\%) while significantly improving MR quality.

At present, there are many methods to reduce the CT radiation dose, including reducing tube voltage, reducing tube current, shortening scanning time, and applying iterative algorithms, amongst others $(16,17)$. However, these methods have rarely been reported in MR. Liu et al. (11) demonstrated that low-dose CT scanning technology $(100 \mathrm{kVp})$ in RA CTA examination could significantly decrease ED by approximately $37.24 \%$. We chose $80 \mathrm{kVp}$ in the present study, further reduced the tube voltage, and reduced ED by approximately $42.3 \%$. Radiation dose is proportional to the square of tube voltage, and tube voltage reduction can greatly reduce radiation dose (17). The low-dose MR group had higher CT attenuation than the normal-dose MR group, as lowering tube voltage can significantly increase CT attenuation. When reducing the tube voltage, the IN can be increased, and the SNR or CNR can be reduced (18). However, our study showed that the IN of the low-dose MR group was lower than that of 
the normal-dose MR group, while SNR and CNR of the low-dose MR group were higher than those of the normaldose MR group. This may be due to the use of the ASiR (GE Healthcare, Waukesha, WI, USA) algorithm. ASiR is based on the mathematic model and statistical iteration of CT data and can effectively decrease IN and improve SNR and CNR (19). Li et al. (20) reported that ASiR could provide clinically acceptable image quality, with an estimated dose reduction in the range of 40-60\%. ATCM technology can automatically adjust the tube current to reduce the radiation dose according to the tissue structural thicknesses and density. It can assess each layer's required $\mathrm{mA}$ values combined with the preset IN and intelligently adjust tube current (21).

An analysis of relevant perioperative indexes was performed. The reasons for the decrease in OT in the MR group can be analyzed as follows: (I) preoperative application of MR helps surgeons correctly formulate operation plans, thus reducing the OT; (II) the surgeons can fully simulate the operation process before surgery so that practice makes perfect; (III) MR facilitates rapid and accurate positioning during the operation. The decrease in WIT may be because the application of MR can accurately identify the renal tumor's responsible vessel by directly clamping the responsible vessel of the tumor, avoiding main RA clamping, achieving zero ischemia (WIT $=0$ min), and significantly reducing EBL. Furthermore, MR can effectively display the APF region before surgery. If widespread APF is judged to be quite serious, the surgeon can directly use $\mathrm{RN}$ and then avoid the increase of EBL. In this study, no significant difference was found among the 3 groups in postoperative Scr, probably due to the compensatory effect of the residual nephron and healthy kidney. We believe that with longer postoperative follow-up and further expansion of sample size, MR has absolute advantages in postoperative renal function recovery.

In our previous study, Li et al. demonstrated that the number of conversions from laparoscopic partial nephrectomy (LPN) to laparoscopic radical nephrectomy (LRN) were $2 / 50(4 \%)$ and $4 / 50(8 \%)$ for the MR group and the non-MR group, respectively (3). However, in the present study, we found that the number of conversions from LPN to LRN were 15/50 (30\%), 13/50 (26\%), and 21/50 (42\%) for Group A, Group B, and Group $\mathrm{C}$, respectively. Firstly, the focus of the application of MR technology in the two studies was different. In the previous study, we enrolled patients who could undergo
NSS thorough preoperative discussion. The purpose was to reduce the difficulty of operation, shorten the OT, and reduce the incidence of forced conversion from LPN to LRN. In the present study, we targeted patients with special conditions (such as the absence of kidney on the non-tumor side and severely impaired renal function on the nontumor side). To avoid further damage to the renal function of these patients, NSS had to be the first choice. It is very difficult for these patients to undergo NSS based on CT images and preoperative discussion. For these patients, due to MR technology, the success rate of NSS was effectively improved.

The reason why the LPN conversion rate to LRN in the MR group was lower than that in the non-MR group may be because, firstly, preoperatively, MR can help formulate an accurate operation plan assessment of surgical risk. During surgery, MR can be used to display the renal tumor in real-time, which helps locate the renal tumor quickly and accurately and ensure the smooth implementation of NSS. Secondly, because MR can fully display the APF area around the renal tumor, surgeons can judge the severity of APF before the operation. If the APF area is wide and serious, it will be more difficult to separate the renal tumor and kidney. Surgeons can consider RN directly to avoid the increase in OT and blood loss; thus, the conversion rate of LPN to LRN decreased.

Sticky fat is much more of a challenge in the study locality than in other regions. We believe that this is due to the anatomical characteristics of the kidney and the surgical method. When the surgeon performs NSS, the first step is to dissect the fat around the kidney from the kidney, while other operations do not have this procedure. When APF occurs, it can increase the difficulty of surgically dissecting the kidney tumor (22). If dissection is forced, it can cause a tear of the renal capsule. It is difficult to suture the wound again after the operation, forcing transfer to RN. At the same time, patients with APF can have reduced operation space and increased difficulty of operation. Although the overall incidence of APF is not very high, Kawamura et al. reported that the incidence of APF in Asian patients with kidney cancer is approximately $17 \%$ (23), but for patients with severe APF, it is a significant challenge for surgeons to perform NSS surgery. In this study, patients with moderate or severe MAP scores (MAP score $>2$ ) (6) were selected for enrollment, which better reflects the important value of MR technology in NSS.

MR is based on CT DICOM data. The quality of CT data directly affects the quality of MR. Our study proved 
that the CT data of the low-dose MR group was better than the normal-dose MR group (the CNR and SNR of the low-dose MR group were higher than those of the normaldose MR group). Therefore, under the same technical conditions, the subjective scores of MR quality in the lowdose MR group were significantly higher than those of the normal-dose MR group. Furthermore, there was no difference in MR build procedure and reconstruction time between the low-dose MR group and the normal-dose MR group. The cost of low-dose CT scanning in most hospitals is equal to that of normal-dose CT scanning, so there is no additional cost for the low-dose MR group.

This study has several limitations. First, the study sample size is small. Second, we did not adopt a different percentage ASiR algorithm to compare the quality of MR. We merely chose $50 \%$ ASiR for optimization according to experience or previous literature. Third, there was no effective grouping of MR applications between APF and non-APF patients. Fourth, this study lacks subjective scores of MR quality by surgeons of different seniority. Finally, real-time $M R$ automatic updates during surgery will be resolved with the development of artificial intelligence and the $5 \mathrm{G}$ network. These limitations will be further discussed in future research.

\section{Conclusions}

Low-dose CT technology can be effectively applied to MR optimization, reducing ED and improving MR quality. Optimized MR can significantly increase the cases of successful NSS and improve perioperative indexes.

\section{Acknowledgments}

The authors would like to thank all the editors and reviewers of QIMS.

Funding: This study was supported by the National Clinical Key Specialist Military Construction Project of China, the Clinical Medical Imaging Center Project of Jiangsu Province, China (grant YXZXA2016007), and the National Key Research and Development Program of China (2017YFC0113404).

\section{Footnote}

Conflicts of Interest: All authors have completed the ICMJE uniform disclosure form (available at http://dx.doi. org/10.21037/qims-20-956). The authors have no conflicts of interest to declare.

Ethical Statement: This study was performed in accordance with the Declaration of Helsinki and was approved by the Ethics Committee of the Jinling Hospital, Medical School of Nanjing University. All patients gave informed consent to participate in the study before examination.

Open Access Statement: This is an Open Access article distributed in accordance with the Creative Commons Attribution-NonCommercial-NoDerivs 4.0 International License (CC BY-NC-ND 4.0), which permits the noncommercial replication and distribution of the article with the strict proviso that no changes or edits are made and the original work is properly cited (including links to both the formal publication through the relevant DOI and the license). See: https://creativecommons.org/licenses/by-nc-nd/4.0/.

\section{References}

1. Gofrit ON, Shapiro A, Kovalski N, Landau EH, Shenfeld OZ, Pode D. Renal cell carcinoma: evaluation of the 1997 TNM system and recommendations for follow-up after surgery. Eur Urol 2001;39:669-74; discussion 675.

2. Cai Y, Li HZ, Zhang YS. Comparison of Partial and Radical Laparascopic Nephrectomy: Long-Term Outcomes for Clinical T1b Renal Cell Carcinoma. Urol J 2018;15:16-20.

3. Li G, Dong J, Wang J, Cao D, Zhang X, Cao Z, Lu G. The clinical application value of mixed-reality-assisted surgical navigation for laparoscopic nephrectomy. Cancer Med 2020;9:5480-9.

4. Saito Y, Sugimoto M, Imura S, Morine Y, Ikemoto T, Iwahashi S, Yamada S, Shimada M. Intraoperative 3D Hologram Support With Mixed Reality Techniques in Liver Surgery. Ann Surg 2020;271:e4-e7.

5. Huang Q, Gu L, Zhu J, Peng C, Du S, Liu Q, Chen J, Wang B, Fan Y, Gao Y, Fam X, Wang H, Liu F, Guo A, Li $\mathrm{H}$, Zhang $\mathrm{X}$, Ma X. A three-dimensional, anatomy-based nephrometry score to guide nephron-sparing surgery for renal sinus tumors. Cancer 2020;126 Suppl 9:2062-72.

6. Davidiuk AJ, Parker AS, Thomas CS, Leibovich BC, Castle EP, Heckman MG, Custer K, Thiel DD. Mayo adhesive probability score: an accurate image-based scoring system to predict adherent perinephric fat in partial nephrectomy. Eur Urol 2014;66:1165-71.

7. Fushima K, Kobayashi M. Mixed-reality simulation for orthognathic surgery. Maxillofac Plast Reconstr Surg 
2016;38:13.

8. Journy N, Ancelet S, Rehel JL, Mezzarobba M, Aubert B, Laurier D, Bernier MO. Predicted cancer risks induced by computed tomography examinations during childhood, by a quantitative risk assessment approach. Radiat Environ Biophys 2014;53:39-54.

9. Vega F, Múgica MV, Argíz L, Bazire R, Belver MT, Friera A, Blanco C. Protocol to prevent contrast-induced nephropathy in parenteral challenge tests for allergy evaluation of hypersensitivity reactions to iodinated contrast media. Clin Exp Allergy 2020;50:1200-3.

10. Kutikov A, Uzzo RG. The R.E.N.A.L. nephrometry score: a comprehensive standardized system for quantitating renal tumor size, location and depth. J Urol 2009;182:844-53.

11. Liu S, Li W, Shi H, Sheng H, Fan J, He J, Sun H. LowDose Scanning Technology Combined with LowConcentration Contrast Material in Renal Computed Tomography Angiography (CTA): A Preliminary Study. Med Sci Monit 2017;23:4351-9.

12. Zemedkun M, LaBounty TM, Bergman G, Wong SC, Lin FY, Reynolds D, Gomez M, Dunning AM, Leipsic J, Min JK. Effectiveness of a low contrast load CT angiography protocol in octogenarians and nonagenarians being evaluated for transcatheter aortic valve replacement. Clin Imaging 2015;39:815-9.

13. McJunkin JL, Jiramongkolchai P, Chung W, Southworth M, Durakovic N, Buchman CA, Silva JR. Development of a Mixed Reality Platform for Lateral Skull Base Anatomy. Otol Neurotol 2018;39:e1137-42.

14. Gill MK, Vijayananthan A, Kumar G, Jayarani K, Ng $\mathrm{KH}$, Sun Z. Use of $100 \mathrm{kV}$ versus $120 \mathrm{kV}$ in computed tomography pulmonary angiography in the detection of pulmonary embolism: effect on radiation dose and image quality. Quant Imaging Med Surg 2015;5:524-33.

15. Shirk JD, Thiel DD, Wallen EM, Linehan JM, White WM, Badani KK, Porter JR. Effect of 3-Dimensional Virtual Reality Models for Surgical Planning of Robotic-
Assisted Partial Nephrectomy on Surgical Outcomes: A Randomized Clinical Trial. JAMA Netw Open 2019;2:e1911598.

16. Aldosari S, Jansen S, Sun Z. Optimization of computed tomography pulmonary angiography protocols using $3 \mathrm{D}$ printed model with simulation of pulmonary embolism. Quant Imaging Med Surg 2019;9:53-62.

17. Leschka S, Stolzmann P, Schmid FT, Scheffel H, Stinn B, Marincek B, Alkadhi H, Wildermuth S. Low kilovoltage cardiac dual-source CT: attenuation, noise, and radiation dose. Eur Radiol 2008;18:1809-17.

18. Liu Y, Liu A, Liu L, Tian S, Liu J, Pu R, Fang X, Liu $\mathrm{X}$, Yuan G. Feasibility of spectral imaging with lowconcentration contrast medium in abdominal CT angiography of obese patients. Int J Clin Pract 2016;70 Suppl 9B:B37-43.

19. Almutairi A, Al Safran Z, AlZaabi SA, Sun Z. Dual energy CT angiography in peripheral arterial stents: optimal scanning protocols with regard to image quality and radiation dose. Quant Imaging Med Surg 2017;7:520-31.

20. Li X, Shu H, Zhang Y, Li X, Song J, Du J, Qian Y, Liu B, Yu Y. Low-dose CT with adaptive statistical iterative reconstruction for evaluation of urinary stone. Oncotarget 2018;9:20103-11.

21. Papadakis AE, Damilakis J. Evaluation of an organbased tube current modulation tool in pediatric CT examinations. Eur Radiol 2020;30:5728-37.

22. Li G, Huang W, Xu Q, Dong J, Cao Z, Wang D, Zou M, Lu G. Application value of dual-energy computed tomography spectrum curve combined with clinical risk factors in predicting adherent perinephric fat. Quant Imaging Med Surg 2019;9:1421-8.

23. Kawamura N, Saito K, Inoue M, Ito M, Kijima T, Yoshida S, Yokoyama M, Ishioka J, Matsuoka Y, Kihara K, Fujii Y. Adherent Perinephric Fat in Asian Patients: Predictors and Impact on Perioperative Outcomes of Partial Nephrectomy. Urol Int 2018;101:437-42.
Cite this article as: Li G, Cao Z, Wang J, Zhang X, Zhang L, Dong J, Lu G. Mixed reality models based on lowdose computed tomography technology in nephron-sparing surgery are better than models based on normal-dose computed tomography. Quant Imaging Med Surg 2021;11(6):2658-2668. doi: 10.21037/qims-20-956 